\title{
LEIOMIOMA DE VEJIGA. ANÁLISIS DE AGREGACIÓN DE 90 CASOS
}

\author{
M. SILVA-RAMOS, P. MASSÓ, R. VERSOS, J. SOARES, A. PIMENTA \\ Serviço de Urologia. Hospital Geral de Santo António. Porto. Portugal. \\ Actas Urol Esp. 27 (8): 581-586, 2003
}

\begin{abstract}
RESUMEN
LEIOMIOMA DE VEJIGA. ANÁLISIS DE AGREGACIÓN DE 90 CASOS

INTRODUCCIÓN: Los leiomiomas de vejiga (LV) son tumores raros. La mayoría de las publicaciones relativas a estos tumores describen casos aislados, no permitiendo evaluar estrategias diagnósticas y terapéuticas.

MATERIAL Y MÉTODO: Realizamos un análisis de agregación de 90 casos de LV descritos en la literatura internacional.

RESULTADOS: La edad media al diagnóstico fue de 45,3 años (19-85 años), 68 (75,6\%) eran mujeres. Los síntomas más frecuentes eran de almacenamiento (50\%) seguidos de los de vaciado (24,4\%). Veinticuatro pacientes $(26,7 \%)$ estaban asintomáticos. Cuarenta y seis tumores $(51,1 \%)$ tenían crecimiento endoluminal, $27(30 \%)$ eran intramurales y $15(16,7 \%)$ extravesicales. A 56 pacientes $(62,2 \%)$ se les realizó una laparotomía, con enucleación en $29(32,2 \%)$, cistectomía parcial en $25(27,8 \%)$ y cistectomía total en $2(2,2 \%)$. Veintisiete $(30 \%)$ fueron sometidos a resección transuretral y 5 pacientes $(5,6 \%)$ a una enucleación transvaginal. Dos pacientes no fueron sometidos a ningún tratamiento. Fueron descritas 3 recidivas y una fístula vesicovaginal como única complicación.

CONCLUSIONES: Aunque el LV sea un tumor raro, en el estudio de una neoformación vesical, los estudios de imagen, pueden hacer sospechar este diagnóstico. El tratamiento quirúrgico tiene una alta tasa de éxito. En la mayoría de los casos, la resección transuretral o la enucleación es suficiente, evitando así una cirugía más iatrogénica.
\end{abstract}

PALABRAS CLAVE: Vejiga. Leiomioma. Diagnóstico. Tratamiento. Meta-analisis.

\section{ABSTRACT}

BLADDER LEIOMYOMA. A POOLED ANALYSIS OF 90 CASES

INTRODUCTION: Bladder leiomyomas (BL) are rare. Most publications regarding these tumours are reports of isolated cases; therefore they don't allow an evaluation of diagnostic and treatment procedures.

MATERIAL \& METHODS: We preformed a pooled analysis of 90 cases of BL reported in the literature.

RESULTS: Mean age was 45.3 (19 to 85 years), 68 (75.6\%) were women. Filling symptoms were the most frequently reported $(50 \%)$, followed by voiding symptoms $(24.4 \%)$. Twenty four patients $(26.7 \%)$ were asymptomatic. Tumours were endoluminal in 46 patients (51.1\%), intramural in 27 (30\%) and extravesical in $15(16.7 \%)$. A laparotomy was preformed in 56 patients $(62.2 \%)$, with enucleation in 29 (32.2\%), partial cystectomy in $25(27.8 \%)$ and total cystectomy in $2(2.2 \%)$. A transurethral resection was preformed in $27(30 \%)$ and a transvaginal resection in $5(5.6 \%)$. Two patients underwent conservative treatment. In 3 cases there were reports of recurrence and one patient got a vesicovaginal fistula.

CONCLUSIONS: Although BL are rare, when evaluating bladder tumours, imaging techniques can make suspect of this neoplasm. Surgical treatment of these tumours has a very high success rate. Usually an enucleation or a transurethral resection is sufficient to render the patient tumour free, avoiding a more iatrogenic surgery.

KEY WORDS: Bladder. Leiomyoma. Diagnostic. Treatment. Meta-analisys. 
$\mathrm{E}^{1}$ leiomioma de vejiga (LV) es un tumor mesenquimatoso benigno que deriva del tejido muscular liso. Es un tumor raro que corresponde a menos de $1 \%$ de los tumores vesicales ${ }^{1}$. Apenas existen 20 casos descritos antes de 1900. En 1929 De Barne-Lagrade recogió 36 casos en la literatura $^{2}$. Más recientemente, Goluboff ha hecho una revisión de 37 casos de leiomioma publicados en la literatura inglesa ${ }^{3}$ y Cornebele describió 23 casos de leiomioma de vejiga y uretra observados en la clínica Mayo ${ }^{4}$. Con todo, la mayoría de las publicaciones sobre LV se refieren a casos aislados, que no permiten hacer una evaluación sobre las diferentes estrategias de abordaje de estos pacientes. Puesto esto, hicimos una revisión de la literatura internacional con el objetivo de establecer unas recomendaciones para el diagnóstico y tratamiento de estos tumores.

\section{MATERIAL Y MÉTODOS}

Hemos recogido 62 artículos de la literatura internacional $^{1-62}$, describiendo un total de 90 casos de LV. Todos ellos fueron analizados en cuanto a edad, sexo, presentación clínica, procedimientos diagnósticos, localización, tratamiento y complicaciones.

\section{RESULTADOS}

De los 90 pacientes estudiados, 68 (75,6\%) eran de sexo femenino. Las edades de presentación variarán entre los 19 y 85 años (media 45,3 años). Los tumores eran únicos a excepción de dos casos en que coexistían con otro leiomioma localizado en la uretra. Los sintomas de presentación más frecuentes eran los de almacenamiento (50\%), como polaquiuria, urgencia y dolor suprapúbico (Tabla I). Veintidós pacientes $(24,4 \%)$ presentaban síntomas de vaciado como disuria, disminución de calibre miccional, tenesmo y en tres pacientes retención urinaria aguda. Hematuria macroscópica fue encontrada en 18 casos (20\%), y 6 de los pacientes $(6,7 \%)$ referían lumbalgia relacionada con la obstrucción ureteral causada por el leiomioma. En 27 (30\%) fue encontrada una masa palpable en la exploración bimanual. Veinticuatro pacientes $(26,7 \%)$ estaban asintomáticos en el momento del diagnóstico, siendo la neoformación descubierta durante un examen ecográfico en 14 pacientes (15,5\%), en examen ginecoló-

\section{TABLA I}

CLÍNICA DE PRESENTACIÓN

\begin{tabular}{||l|c|}
\hline Clínica & № pacientes (\%) \\
\hline Asintomáticos & $24(26,7)$ \\
\hline Sintomas de almacenamiento & $45(50)$ \\
\hline Sintomas de vaciamiento & $22(24,4)$ \\
\hline Retención urinaria aguda & $3(3,3)$ \\
\hline Hematuria & $18(20)$ \\
\hline Lumbalgia & $6(6,7)$ \\
\hline Insuficiencia renal & $2(2,2)$ \\
\hline Masa palpable & $27(30)$ \\
\hline
\end{tabular}

gico en $6(6,7 \%)$ y en urografía intravenosa (UIV) en $2(2,2 \%)$. Tan sólo uno de los enfermos asintomáticos era de sexo masculino.

Se realizó cistoscopia en 82 pacientes $(91,1 \%)$, mostrando lesión en 77 (93,9\%) (Tabla II). El examen resultó negativo en 2 casos con tumor intramural y en 3 con tumor extravesical. Sesenta y ocho pacientes $(75,6 \%)$ realizaron UIV que mostró lesión en 58 (85,2\%), la mitad de los tumores de la pared anterior no fueron identificados. Fueron realizadas ecografía, tomografía axial computerizada (TAC) y resonancia magnética nuclear (RMN) en 58 (64,4\%), 36 (40\%) y 13 (14,4\%) pacientes respectivamente, en todos estos exámenes fue detectada lesión.

En 28 pacientes $(31,8 \%)$ fue efectuada biopsia antes del tratamiento definitivo (Tabla III). Las biopsias realizadas con aguja "True-cut" y las realizadas a través de resección endoscópica resultaron diagnósticas en todos los casos. Sin embargo, las biopsias endoscópicas a frío fueron falsamente negativas en el 50\% de los casos. Las biopsias aspirativas resultaron ser las que ofrecían menos

\section{TABLA II}

EXÁMENES COMPLEMENTARIOS DE DIAGNÓSTICO

\begin{tabular}{|l|c|c|}
\hline Examen & № pacientes (\%) & Observada lesión (\%) \\
\hline Cistoscopia & $80(90,9)$ & 93,9 \\
\hline Urografia & $68(77,3)$ & 85,2 \\
\hline Ecografia & $57(64,8)$ & 100 \\
\hline TAC & $35(39,8)$ & 100 \\
\hline RMN & $13(14,8)$ & 100 \\
\hline
\end{tabular}


TABLA III

TIPO DE BIOPSIA

\begin{tabular}{|l|c|c|}
\hline Tipo de Biopsia & No de pacientes & \% positiva \\
\hline Resección endoscópica & 8 & 100 \\
\hline Biopsia aspirativa & 8 & 12,5 \\
\hline $\begin{array}{l}\text { Biopsia endoscópica } \\
\text { a frio }\end{array}$ & 6 & 50 \\
\hline Biopsia “true-cut” & 5 & 100 \\
\hline No especificada & 1 & - \\
\hline
\end{tabular}

información, en 4 pacientes no fue conseguido producto con células, en otros 3 fueron observadas células de músculo liso, que aisladamente no permitían dar el diagnóstico de leiomioma y apenas en 1 fue sugerido el diagnóstico de LV.

El tumor era endoluminal en 46 pacientes $(51,1 \%)$, predominantemente intramural en 27 (30\%) y extravesical en 15 (16,7\%). En dos casos no existian datos suficientes para la clasificación. La zona de vejiga más frecuentemente afectada era el cuello (Tabla IV). Los tumores tenían un tamaño que variaba entre 1 y $25 \mathrm{~cm}$ (media-5,3 cm). Siendo los de crecimiento extravesical los más voluminosos (Tabla V).

A $56(62,2 \%)$ se les realizó una laparotomía (Tabla VI), con enucleación del tumor en 29, cistectomía parcial en 25 y cistectomía total en 2 . Al mismo tiempo que el tratamiento de exéresis, fueron realizadas 5 ureteroneocistostomías, 2 nefrouretectomías por anulación funcional renal y una uretrectomía por extensión del leiomioma hacia uretra. En 27 casos (30\%) fue realizada resección transuretral (RTU) y en 5 pacientes $(5,6 \%)$ se realizó enucleación vía transvaginal de tumores que se localizaban a nivel de cuello vesical. En 2

\section{TABLA IV}

ZONA DE VEJIGA AFECTADA

\begin{tabular}{|l|c|}
\hline Zona & № de pacientes* (\%) \\
\hline Cuello & $31(34,4)$ \\
\hline Paredes laterales & $27(30)$ \\
\hline Pared posterior & $17(18,9)$ \\
\hline Pared anterior & $8(8,9)$ \\
\hline Cúpula & $5(5,6)$ \\
\hline
\end{tabular}

*En 2 casos no había descripción de la zona afectada.
TABLA V

TAMAÑO DEL TUMOR Y SU RELACIÓN CON LA LOCALIZACIÓN Y TRATAMIENTO REALIZADO

\begin{tabular}{||l|c|}
\hline Localización & $\begin{array}{c}\text { Tamaño medio } \pm \\
\text { desvío padrón }\end{array}$ \\
\hline Endoluminal & \\
Intramural & $4,6 \pm 3,6$ \\
Extravesical & $5,7 \pm 4,3$ \\
\hline Tratamiento & $6,7 \pm 5,2$ \\
\hline Conservador & $3,5 \pm 2,1$ \\
Transvaginal & $4,8 \pm 1,4$ \\
RTU & $3,4 \pm 1,8$ \\
Enucleación por laparotomía & $6,2 \pm 4,9$ \\
Cistectomía parcial & $5,4 \pm 2,2$ \\
Cistectomía total & $15 \pm 14,1$ \\
\hline
\end{tabular}

pacientes no se realizó tratamiento quirúrgico, en estos casos se les hizo biopsias que confirmaron la benignidad de la lesión.

En 45 casos existía información relativa al seguimiento de estos enfermos, que varió entre 2 y 244 meses (media-46 meses). En ningún enfermo se describió degeneración maligna del tumor. Apenas hay referencia a una complicación, una fístula vesicovaginal después de RTU. Tres enfermos presentaron recidiva, 2 tras enucleación vía vaginal y 1 tras RTU. Estos pacientes han sido posteriormente reoperados con suceso, uno con uretrectomía y resección parcial del cuello vesical, otro por enucleación vía vaginal y otro por cistectomía parcial.

\section{DISCUSIÓN}

Los leiomiomas de vejiga, aunque raros, son los tumores mesenquimatosos benignos más frecuentes de la vejiga. Con el aumento del número de exámenes ecográficos y ginecológicos de rutina, es de prever un aumento de diagnósticos de leiomioma en enfermos asintomáticos. La mayoría de casos publicados era de mujeres con edades entre los 20 y 60 años (85\%), datos que coinciden con la revisión de Goluboff y colaboradores. Esta predominancia de mujeres es más evidente en los casos asintomáticos $(95,8 \%)$, lo que podrá estar relacionado con el hecho de que las muje- 
TRATAMIENTO SEGÚN LA LOCALIZACIÓN

\begin{tabular}{|c|c|c|c|c|c|}
\hline \multirow{2}{*}{\multicolumn{2}{|c|}{ Tratamiento }} & \multicolumn{4}{|c|}{ № de pacientes } \\
\hline & & Endoluminal & Intramural & Extravesical & Total* (\%) \\
\hline \multicolumn{2}{|l|}{ Conservador } & 1 & 1 & 0 & $2(2,2)$ \\
\hline \multicolumn{2}{|c|}{ Exéresis transvaginal } & 0 & 2 & 3 & $5(5,6)$ \\
\hline \multicolumn{2}{|l|}{ RTU } & 23 & 3 & 0 & $27(30)$ \\
\hline \multirow[t]{3}{*}{ Laparotomía } & Enucleación & 14 & 6 & 8 & $29(32,2)$ \\
\hline & Cistectomía parcial & 7 & 14 & 4 & $25(27,8)$ \\
\hline & Cistectomía total & 1 & 1 & 0 & $2(2,2 \%)$ \\
\hline
\end{tabular}

*En 2 casos no había información suficiente para localizar el tumor.

res realizan más exámenes ecográficos que los hombres y de que tienen la pared de la vejiga accesible al examen ginecológico.

Los síntomas más frecuentes fueron los de almacenamiento, lo que difiere de la revisión de Goloboff y cols. en que el $49 \%$ de los enfermos presentaban sintomas obstructivos. El tipo de síntomas de almacenamiento o de vaciado no estaba relacionado con la localización intra o extravesical del tumor, pero si con la zona de la vejiga afectada. La mayoría de pacientes con síntomas de vaciado (59\%) tenía tumor a nivel de cuello. Esta localización debido a que puede causar obstrucción de la uretra y de los uréteres está también asociada a secuelas importantes, 2 pacientes tenían IRC y otro fue nefrectomizado por anulación funcional de dicho riñón.

En nuestra revisión la TAC y la UIV fueron los exámenes diagnósticos que aportaron menos información. La UIV aunque usada con frecuencia, fue menos sensible y específica que la ECO, TAC o RMN. Por otro lado, la TAC no permite caracterizar la naturaleza líquida o sólida de la lesión y comparativamente con ECO y RMN parece delimitar con menor exactitud su relación con la pared vesical.

La ecografía es un examen que nos da mucha información y debe ser usado en primera línea del estudio de estos pacientes. El aspecto ecográfico de estos tumores, es de una masa de ecogeneidad homogénea, bien delimitada y de forma más o menos circular. Si se trata de un tumor endoluminal, la mucosa se presenta como un halo hiperecogenio que recubre la masa. Estas características nos permiten sospechar de una neoformación no epitelial. Cuando tiene localización extravesical y en la pared posterior, la ecografía endovaginal nos da mayor definición ${ }^{5}$.

Los leiomiomas tienen un aspecto característico en la RMN. Se presentan como nódulos de baja intensidad, de superficie regular y con límites bien definidos permitiendo distinguirlos fácilmente de otras estructuras ${ }^{6}$.

La cistoscopia fue el examen realizado con más frecuencia, presentándose el tumor como una masa redondeada cubierta por mucosa regular. Los 5 exámenes falsos negativos, eran de enfermos con tumores de dimensiones reducidas, sin crecimiento endoluminal.

A pesar de todo, no existe ningún medio de imagen que permita distinguir con seguridad un leiomioma de un leiomiosarcoma, por lo que varios autores intentaron caracterizar histológicamente la lesión antes de decidir la terapéutica a aplicar. Las punciones aspirativas dan poca información, por lo que cuando las lesiones son extravesicales sugerimos toma de tejido mediante aguja "True-cut". Si es posible realizar biopsia por RTU, debe efectuarse, ya que se trata de un procedimiento diagnóstico y terapéutico al mismo tiempo. La biopsia endoscópica a frío ofrece muchos falsos negativos, dado que las pinzas de biopsia cogen esencialmente mucosa, estando la capa muscular poco representada.

Los tumores endovesicales se tratan de forma adecuada por RTU, siendo el factor limitante de 
esta técnica el tamaño del tumor. Así, cuando estos tumores son voluminosos la resección a cielo abierto evita varias RTUs. Para los tumores intramurales y extravesicales, la enucleación parece ser igual de eficaz que la cistectomía parcial. La mayoría de los autores refiere que estos tumores son fácilmente enucleados ${ }^{7-9}$ pudiendo ser evitada una cirugía más agresiva.

De los 5 enfermos a los que se les realizó enucleación por vía vaginal, 2 presentaron recidiva, probablemente relacionada con la dificultad de exposición en el campo operatorio y el sangrado fácil. Pensamos que esta vía debe ser usada con cuidado, pero que continua siendo ideal para tumores palpables por vía vaginal, localizados cerca de la uretra.

Aunque el LV sea un tumor infrecuente, en el estudio de una neoformación vesical, los estudios de imagen, sobretodo la ecografía y la RMN, pueden hacernos sospechar este diagnóstico. Con todo, estos medios no permiten excluir malignidad con seguridad. El tratamiento de estos tumores es simple y se resuelve con éxito en la mayor parte de los casos, a través de RTU o enucleación, evitando técnicas quirúrgicas más agresivas.

\section{REFERENCIAS}

1. BLASCO CASARES FJ, SACRISTÁN SANFILIPE J, IBARZ SERVIO L, BATALLA CADIRA JL, RUIZ MARCELLÁN FJ.: Características del leiomioma vesical en nuestro medio. Arch Esp Urol 1995; 48: 987-990.

2. CAMPBELL EW, GISLASON JG.: Benign mesothelial tumors of the urinary bladder: review of the literatura and a report of a case of leiomyoma. J Urol 1953; 70: 733-741.

3. GOLUBOFF ET, O'TOOL K, SAWCZUK IS.: Leiomyoma of the bladder: report of a case and review of the literature. Urology 1994; 43: 238-341.

4. CORNELLA JL, LARSON TR, LEE RA, MAGRINA JF, KAMMERER-DOAK D.: Leiomyoma of the female urethra and bladder: report of twenty-three patients and review of the literature. Am J Obstet Gynecol 1997; 176: 1.278-1.285.

5. FERNÁNDEZ FERNÁNDEZ A, MAYAYO DEHESA T.: Leiomyoma of the urinary bladder floor: diagnosis by transvaginal ultrasound. Urol Int 1992; 48: 99-101.

6. SUNDARAM CP, RAWAL A, SALTZMAN B.: Characteristics of bladder leiomyoma as noted on magnetic resonance imaging. Urology 1998; 52: 1.1421.143.

7. DELAHUNT B, NACEY JN, FERGUSON AF.: Giant sclerosing leiomyoma of bladder presenting as chronic renal failure. Urology 1991; 37: 270-272.
8. TORRUBIA ROMERO FJ, MONTAÑÉS MEDINA P, SÁNCHEZ GONZÁLEZ M, SÁNCHEZ GÓMEZ E.: Leiomioma vesical. Arch Esp Urol 1988; 41: 17-21.

9. BROESSNER C, KLINGLER CH, BAYER G, PYCHA A, KUBER W.: A 3,500 gram leiomyoma of the bladder: case report on a 3-year follow-up surgical enucleation. Urol Int 1998; 61: 175-177.

10. SILVA-RAMOS M, VERSOS R, CADILHE P, SOARES J, PIMENTA A.: Bladder leiomyoma, presenting as a cystitis-like syndrome. Internet $J$ Urol; en prensa.

11. OH-OKA H, GOTOH A, HANIOKA K, OKADA H.: Leiomyoma of the urinary bladder causing tamponade. Scand J Urol Nephrol 1998; 32: 420-421.

12. ELIA G, JAMES W, BALLARD CA, BERGMAN A.: Diagnostic considerations in coexisting bladder and urethral leiomyomata. A case report. $J$ Reprod Med 1995; 40: 670-672.

13. SUGI M, SOUMA T, YAMAMOTO S, HATAYAMA T.: Retrovesical leiomyoma: a case report. Hinyokika Kiyo 1996; 42: 687-689.

14. NISHIMURA K, OGAWA O, YOSHIMURA N, NAKAGAWA T.: A case of leiomyoma of the urinary bladder in a woman with the chief complaint of total urinary retension. Hinyokika Kiyo 1984; 30: 41-48.

15. MIURA H, SEGUCHI T, SUGAO H et al.: Leiomyoma of urinary bladder: report of a case. Hinyokika Kiyo 1990; 36: 609-612.

16. NAGANUMA T, YASUMOTO R, KAWANO $\mathrm{M}$ et al.: Leiomyoma of the bladder: report of two cases. Hinyokika Kiyo 1998; 44: 833-837.

17. RIBEIRO JC, SOUSA L, SILVA C, GARCÍA P, VILAMENDES, SANTOS A.: Leiomioma da bexiga simulando neoplasia do úraco. Acta Urol Portuguesa; en prensa.

18. BAZEED MA, ABOULENIEN H.: Leiomyoma of the bladder causing urethral and ureteral obstruction: a case report. J Urol 1988; 140: 143-144.

19. LAKE MH, KOSSOW AS, BOKINSKY G.: Leiomyoma of the bladder and urethra. J Urol 1981; 125: 742743.

20. KIRSH EJ, SUDAKOFF G, STEINBERG GD, STRAUS FH, GERBER GS.: Leiomyoma of the bladder causing ureteral and bladder outlet obstruction. J Urol 1997; 157: 1843.

21. MARTÍNEZ CAMPOS T, VICENTE CARRETERO R, GUERRO POLO JA.: Leiomioma vesical: un nuevo caso a la casuística existente. Actas Urol Esp 1995; 19: $481-483$.

22. LOH CS, RICHARDS CJ, JENKINS BJ.: Bladder neck leiomyoma causing obstructive renal failure. Scand J Urol Nephrol 1996; 30: 495-496.

23. PÉREZ FMA, SALOM MG, NUEZ PC et al.: Leiomioma vesical. Revisión y presentación de un caso. Arch Esp Urol 1995; 48: 1.035-1.037.

24. FÚNEZ FA, CIDRE MJ, FERNÁNDEZ MF, GUERRA NC, ROCA CC.: Leiomioma vesical de crecimiento extramural. Una causa poco frecuente de dolor pélvico. Arch Esp Urol 1999; 52: 800-801.

25. SUHLLER A, MASSON J CL, PAGES CL, DOUILLET P.: Léiomyomes et fibromes vésicaux- à propos de huit cas. Ann Urol 1994; 28: 28-32. 
26. GOLDBERG J, AMARO JL,VERCESI L, TRINDADE JCS.: Le leiomyome de la vessie. J Urol (Paris) 1994; 100: 49-50.

27. MARC B, LEBON E, MAZEMAN E.: Le léiomyome de vessie- à propos d' un cas. J Urol (Paris) 1990; 96: $45-47$.

28. GRUENWALD I, STEIN A, ABRAMOVICI H, LURIE A.: A case of leiomyoma of the bladder during pregnancy. Br J Urol 1997; 79: 647.

29. MARTÍN-MARQUINA A, RODRÍGUEZ-RUBIO FI, ABAD JI et al.: Leiomioma vesical: aportacion de un nuevo caso. Actas Urol Esp 1994; 18: 701-702.

30. HERRANZ AMO F, VERDÚ TARTAJO F, HERNÁNDEZ FERNÁNDEZ C, DIEZ CORDERO JM, DIEZ YANGUAS JM.: Leiomioma vesical. Actas Urol Esp 1988; 12: 65-68.

31. FERNÁNDEZ FERNÁNDEZ A, MAGANTO PAVÓN E, MAYAYO DEHESA T, ROMERO MAROTO J, ESCUDERO BARRILERO A.: Técnicas de estudio morfológico en el leiomioma de suelo vesical en la mujer. Actas Urol Esp 1989; 13: 213-216.

32. RUBIO MUÑOZ A, BONO ARIÑO A, BERNÉ MANERO JM et al.: Leiomioma vesical. Arch Esp Urol 2000; 53: 934-937.

33. NÚÑEZ MORA C, JULVE VILLALTA E, HARDISSON HERNÁEZ D et al.: Leiomioma vesical durante el embarazo. Arch Esp Urol 1999; 52: 510-512.

34. GONZÁLEZ AL, CIFUENTES JLR, MUÑOZ ML.: Leiomioma vesical. Aportación de un nuevo caso. Arch Esp Urol 1991; 44: 1.145-1.147.

35. GÓMEZ VEGAS A, SILMI MOYANO A, FERNÁNDEZ LUCAS C et al.: Leiomiomas de tracto urinario inferior. Arch Esp Urol 1991; 44: 795-798.

36. CERVELL FERRÓN JM, ASENSIO EGEA L, MORENO AVILÉS J, SOLER FERNÁNDEZ J, GARCÍA PÉREZ R.: Leiomioma vesical. Arch Esp Urol 2000; 53: 943-946.

37. CHICHARRO MOLERO JA, BAENA GONZÁLEZ V, QUIÑONERO DÍAZ A, PADILLA LEÓN M, ALLONA MONCADA A.: Leiomioma vesical: un caso de diagnóstico equívoco. Arch Esp Urol 1987; 40: 678-680.

38. OJEA CALVO A, NÚÑEZ LÓPEZ A, ALONSO RODRIGO A et al.: Leiomioma vesical. Actas Urol Esp 2001; 25: 759-763.

39. PUIG-GIRO R, GUAJARDO J, BARCO J et al.: Leiomioma de vejiga causante de una obstrucción unilateral del uréter asociado a leiomiomas uterinos y ovarios poliquísticos. Actas Urol Esp 1990; 14: 302-304

40. RODRÍGUEZ DE LEDESMA VEGA JM, TORROELLA SANTS V, RAMIRES RUZ J, BATLLE MELER G.: Leiomioma vesical. Revisión de la literatura y presentación de un nuevo caso. Arch Esp Urol 2000; 53: 167-170.

41. ABENGOZAR GARCÍA MORENO A, MURILLO MIRAT J, CUERVO PINNA C, RODRÍGUEZ RINCÓN P, CABALLERO GÓMEZ M, SEVILLA ZABALETA M.: Leiomioma vesical: revisión de la literatura y presentación de tres casos clínicos. Actas Urol Esp 1998; 22: 702-706.

42. ULLATE JAIME V, ANGULO CUESTA J, LOIZAGA IRIARTE A, FLORES CORRAL N.: Leiomioma vesical: a propósito de un caso. Arch Esp Urol 1994; 47: 812-814.
43. GALLIZIA G, FRANCONE E.: Un cas de fibro-leiomyome de la paroi vésicale. J Urol Nephrol 1969: 75: 879-881.

44. CUKIER J, BENHAMOU G. J Urol Nephrol 1970; 76: 61-67.

45. FEKKAR H, MOUFID K, JOUAL A, BENNANI S, EL MRINI M, BENJELLOUN S.: Les leiomyomes de la voie excretrice urinaire. Ann Urol 2001; 35: 47-50.

46. KOSKIVUO IO, ALA-OPAS MY.: Leiomyoma of the bladder. Scan J Urol Nephrol 1992; 26: 193-194.

47. VARGAS AD, MÉNDEZ R: Leiomyoma of bladder. Urology 1983; 21: 308-309.

48. KNOLL DL, SEGURA JW, SHEITHAUER BW: Leiomyoma of the bladder. $J$ Urol 1986; 136: 906908.

49. AMANO T, ISHIURA Y, OHKAWA M.: Case report: bladder leiomyoma- Pre-operative diagnosis by tranrectal ultrassonography guided needle biopsy. Clinical Radiology 1996; 51: 664-665.

50. FURUHASHI M, SUGANUMA N.: Recurrent bladder leiomyoma with ovarian steroid hormone receptors. J Urol 2002; 167: 1.399-1.400.

51. HUANG HY, CHEN WJ, SUNG MT, HUANG CC.: Atypical leiomyoma of the urinary bladder. Sc J Urol Nephrol 2001; 36: 231-233.

52. STROHMER H, ROEHLICH M, HAFNER E, MAIER U.: Leiomyoma of the vesicovaginal septum. Arch Gynecol Obstet 2001; 265: 94-95.

53. KABALIN JN, FREIHA FS, NIEBEL JD.: Leiomyoma of bladder: report of 2 cases and demonstration of ultrassonic appearance. Urology 1990; 35: 210-212.

54. SOLOWAY D, SIMON MA, MILIKOWSKI C, SOLOWAY MS.: Epithelioid leiomyoma of the bladder: an unusual cause of voiding symptoms. Urology 1998; 51: 1.037-1.039.

55. ALBERT NE.: Leiomyoma of bladder: preoperative diagnosis by ultrassound. Urology 1981; 17: 486487.

56. JACOBS MA, BAVENDAM T, LEACH GE.: Bladder leiomyoma. Urology 1989; 34: 56-57.

57. BOLLINGER B, MIKKELSEN AL.: Leiomyoma of the urinary bladder. Urol Int 1985; 40: 43-44.

58. PERIMENIS P, SPEAKMAN M.: Incidental presentation of leiomyoma of bladder with carcinoma of the prostate. Int Urol Nephrol 2000; 32: 279-280.

59. VAN REGEMORTER G, GERMEAU F.: Leiomyoma of the bladder. Eur Urol 1984; 10: 210-211.

60. TERAN A, DON GAMBRELL R.: Leiomyoma of the bladder: case report and review of the literature. Int $J$ Fertility 1989; 34: 289-292.

61. BRAMWELL SP, PITTS J, GOUDIE SE, ABEL BJ.: Giant leiomyoma of the bladder. Br J Urol 1987; 60: 178-184.

62. YUSIM IE, NEULANDER EZ, EIDELBERG I, LISMER LJ, KANATI J.: Leiomyoma of the genitourinary tract. Scan J Urol Nephrol 2001; 35: 295-299.

Dr. M. Silva-Ramos

Serviço de Urologia. Hosp. Geral de Santo António Largo Prof. Abel Salazar

4050 Porto (Portugal)

(Trabajo recibido el 2 diciembre de 2002) 